\title{
Evidence for niche adaptation in the genome of the bovine pathogen Streptococcus uberis
}

Philip N Ward ${ }^{1}$, Matthew TG Holden*2, James A Leigh ${ }^{3}$, Nicola Lennard ${ }^{2}$, Alexandra Bignell2, Andy Barron'2, Louise Clark2, Michael A Quail2, John Woodward ${ }^{2}$, Bart G Barrell ${ }^{2}$, Sharon A Egan ${ }^{3}$, Terence R Field 4 , Duncan Maskell5, Michael Kehoe ${ }^{6}$, Christopher G Dowson ${ }^{7}$, Neil Chanter ${ }^{8}$, Adrian M Whatmore7,9, Stephen D Bentley ${ }^{2}$ and Julian Parkhill ${ }^{2}$

\footnotetext{
Address: ${ }^{1}$ Nuffield Department of Clinical Laboratory Sciences, Oxford University, John Radcliffe Hospital, Headington, Oxford, OX3 9DU, UK, 2The Wellcome Trust Sanger Institute, Wellcome Trust Genome Campus, Hinxton, Cambridge, CB10 1SA, UK, ${ }^{3}$ The School of Veterinary Medicine and Science, The University of Nottingham, Sutton Bonington Campus, Sutton Bonington, Leicestershire, LE12 5RD, UK, ${ }^{4}$ Institute for Animal Health, Compton Laboratory, Compton, Newbury, Berks, RG20 7NN, UK, ${ }^{5}$ Dept. of Veterinary Medicine, The University of Cambridge, Cambridge, CB3 OES, UK, 'Institute for Cell and Molecular Biosciences, The Medical School, University of Newcastle upon Tyne, Framlington Place, Newcastle upon Tyne, NE2 4HH, UK, ${ }^{7}$ Department of Biological Sciences, University of Warwick, Coventry, CV4 7AL, UK, ${ }^{8}$ Centre for Preventative Medicine, Animal Health Trust, Newmarket, Suffolk, CB8 7UU, UK and ${ }^{9}$ Veterinary Laboratories Agency, Weybridge, UK

Email: Philip N Ward - Phil.Ward@ndcls.ox.ac.uk; Matthew TG Holden* - mh3@sanger.ac.uk; James A Leigh - James.Leigh@nottingham.ac.uk; Nicola Lennard - nj1@sanger.ac.uk; Alexandra Bignell - al1@sanger.ac.uk; Andy Barron - ab5@sanger.ac.uk; Louise Clark - lnc@sanger.ac.uk; Michael A Quail - maq@sanger.ac.uk; John Woodward - jrw@sanger.ac.uk; Bart G Barrell - barrell@sanger.ac.uk;

Sharon A Egan - Sharon.Egan@nottingham.ac.uk; Terence R Field - terry.field@bbsrc.ac.uk; Duncan Maskell - djm47@cam.ac.uk;

Michael Kehoe-mike.kehoe@ncl.ac.uk; Christopher G Dowson - c.g.dowson@warwick.ac.uk;

Neil Chanter - neilchanter@neilchanterphotography.com; Adrian M Whatmore - a.whatmore@vla.defra.gsi.gov.uk;

Stephen D Bentley - sdb@sanger.ac.uk; Julian Parkhill - parkhill@sanger.ac.uk

* Corresponding author
}

Published: 28 january 2009

BMC Genomics 2009, 10:54 doi:10.1186/147I-2164-10-54
Received: 29 August 2008

Accepted: 28 January 2009

This article is available from: http://www.biomedcentral.com//47I-2/64/10/54

(c) 2009 Ward et al; licensee BioMed Central Ltd.

This is an Open Access article distributed under the terms of the Creative Commons Attribution License (http://creativecommons.org/licenses/by/2.0), which permits unrestricted use, distribution, and reproduction in any medium, provided the original work is properly cited.

\begin{abstract}
Background: Streptococcus uberis, a Gram positive bacterial pathogen responsible for a significant proportion of bovine mastitis in commercial dairy herds, colonises multiple body sites of the cow including the gut, genital tract and mammary gland. Comparative analysis of the complete genome sequence of S. uberis strain 0I40J was undertaken to help elucidate the biology of this effective bovine pathogen.

Results: The genome revealed I,825 predicted coding sequences (CDSs) of which 62 were identified as pseudogenes or gene fragments. Comparisons with related pyogenic streptococci identified a conserved core (40\%) of orthologous CDSs. Intriguingly, S. uberis 0I40J displayed a lower number of mobile genetic elements when compared with other pyogenic streptococci, however bacteriophage-derived islands and a putative genomic island were identified. Comparative genomics analysis revealed most similarity to the genomes of Streptococcus agalactiae and Streptococcus equi subsp. zooepidemicus. In contrast, streptococcal orthologs were not identified for $11 \%$ of the CDSs, indicating either unique retention of ancestral sequence, or acquisition of sequence from alternative sources. Functions including transport, catabolism, regulation and CDSs
\end{abstract}


encoding cell envelope proteins were over-represented in this unique gene set; a limited array of putative virulence CDSs were identified.

Conclusion: S. uberis utilises nutritional flexibility derived from a diversity of metabolic options to successfully occupy a discrete ecological niche. The features observed in S. uberis are strongly suggestive of an opportunistic pathogen adapted to challenging and changing environmental parameters.

\section{Background}

Streptococcus uberis is a gram positive bacterium belonging to family Streptococcaceae, a diverse family of bacteria that encompasses species capable of commensal and/or pathogenic traits. Pathogenic streptococci cause a variety of disease states across a range of animal hosts as well as man. The zoonotic potential of streptococci normally considered pathogenic for animal species has been recently documented for Streptococcus suis [1] and Streptococcus agalactiae [2].

Phylogenetic analysis [3] placed S. uberis within the pyogenic cluster, a large grouping containing the human pathogens Streptococcus pyogenes and Streptococcus dysgalactiae subsp. equisimilis, the zoonotic $S$. agalactiae and a number of animal pathogens occupying diverse ecological niches including S. dysgalactiae, Streptococcus equi, Streptococcus canis and Streptococcus iniae.

S. uberis is commensal at many body sites and has been isolated from the skin, gut, tonsils and genital tract of asymptomatic cattle. Furthermore it can infect the bovine mammary gland and act as a major pathogen of the mammary gland causing the inflammatory disease, mastitis. Infection with $S$. uberis is one of the major causes of bovine mastitis worldwide [4-6] and the most common cause in the UK [7]. Procedures to control bacterial infection of the mammary glands of dairy cattle are based on limiting duration of existing infection and restricting exposure of potentially infectious material from one gland to another. These procedures have resulted in decreased transmission of infections due to certain bacterial species (Staphylococcus aureus, S. agalactiae) but have had little impact on the incidence of infection due to $S$. uberis. The failure of these measures to control intramammary infection due to $S$. uberis implies transmission from additional/alternate sources [8]. Typing of isolates from cases of mastitis also implies that $S$. uberis is not transmitted from reservoirs containing single outbreak strains as multiple bacterial types are often detected within a single herd. S. uberis is often detected in faeces and can also be isolated from the environment (pasture, bedding materials) populated by these animals $[9,10]$. However, survival of $S$. uberis in the environment is limited. A recent report from New Zealand, which operates a pasture-based dairy system where cattle are housed rarely if at all, showed that the organism survived in the environment for less than 4 weeks [11]. This implies that persistence in pasture is dependent on constant reintroduction, probably via faecal contamination. It is, therefore reasonable to conclude that a successful clone of $S$. uberis isolated from a mastitic mammary gland is able to colonise and increase in number within the ruminant gut, survive in environmental niches such as pasture or bedding in sufficient numbers to gain access to the mammary gland where it must replicate and avoid a number of host defence mechanisms. In addition to infection of the lactating mammary gland, $S$. uberis is also able to infect the involuted or dry gland [12]. In this niche the secretion in which the organism replicates and the range of host defences encountered differ markedly from those present during lactation [13].

Epidemiologically, S. uberis strain 0140J, the strain chosen for sequence determination, was placed within a major UK lineage, the clonal complex based around sequence type 5, of an ongoing MLST scheme [14]. As such, strain 0140J represents a typical UK isolate in terms of its ancestry. It is also among the most thoroughly characterised strains [15] that is pathogenic for both the lactating and non-lactating bovine mammary gland. Therefore it was deemed ideally suited to be the first strain of this species to be sequenced. The complete $S$. uberis genome provides insights into host-cell interactions and pathogenesis.

Since the completion of the first streptococcal genome [16] many comparative projects have centred upon the main species pathogenic for humans, namely $S$. agalactiae [17], Streptococcus pneumoniae $[18,19]$ and S. pyogenes $[20,21]$. Such studies have indicated the pairing of significant levels of conserved gene content with considerable gene sequence heterogeneity. Additionally, the proportion and content of such genomes that was attributable to a variety of mobile genetic elements appeared considerable. Comparative genomics has recently enabled the scale of both inter and intra-species horizontal gene transfer to be realised, for example within the oral streptococci [22]. Intriguingly, the gene content of some streptococci also appears to have been augmented from non-streptococcal species with which they co-exist in discrete ecological niches $[23,24]$. It is against such a backdrop that analysis of pathogenic streptococci of veterinary significance can derive added value. The genome sequences of several 
other related streptococcal species, with different host ranges and disease associations are available for comparison. We utilized the genomes of S. equi subsp. zooepidemicus (S. zooepidemicus) [25]; a veterinary pathogen causing lower airway disease, foal pneumonia, endometritis, and abortion in horses, and hemorrhagic streptococcal pneumonia in dogs; and S. pyogenes (alternatively referred to as group A Streptococcus, GAS) [26]; responsible for a diverse number of diseases in humans, including pharyngitis, toxic shock syndrome (TSS), impetigo and scarlet fever, and the post infection sequelae, acute rheumatic fever (ARF). Comparisons with these related pathogens and their virulence determinants highlighted the components of the genome that distinguish it from these species, and genes that are important for the niche-adaptation and virulence of $S$. uberis.

\section{Results and discussion Comparative genomics}

The genome of $S$. uberis $0140 \mathrm{~J}$ consists of a single circular chromosome of 1,852,352 bp (Figure 1), which places it at the lower end of the $1.8 \mathrm{Mb}-2.3 \mathrm{Mb}$ size range of streptococcal genomes sequenced to date. The genome contains 1,825 predicted protein coding sequences (CDSs), 62 of which are pseudogenes or gene fragments (Additional file 1). Comparative genomic analysis with other streptococci by reciprocal FASTA revealed a conserved core of orthologous CDSs (Figure 1); comparisons using representatives of each of the sequenced Streptococcus species identified that $\sim 40 \%$ of $S$. uberis CDSs had orthologous matches in all the streptococcal genomes compared. Supplementing this core were variably distributed orthologues ( $\sim 48 \%$ of the CDSs) that were identified in one or more streptococci, and S. uberis-specific CDSs ( 11\% of the CDSs).

For any one streptococcal species comparison, between $57 \%$ and $72 \%$ of the S. uberis CDSs had orthologue matches, which compared with $58 \%$ for a comparison with Lactococcus lactis subsp. lactis. The highest numbers of orthologue matches were identified in comparisons with pyogenic streptococci, while more taxonomically divergent species yielded lower numbers of orthologous matches. Comparison of the structure of the S. uberis 0140J chromosome with other streptococci revealed the greatest overall conservation with $S$. zooepidemicus and $S$. pyogenes (Figure 2A).

Most of the conserved regions of these genomes appear to be co-linear, interspersed with regions that appear to be translocated and inverted. Of the currently sequenced streptococci, these two pyogenic species are also the most closely related to $S$. uberis as defined by $16 \mathrm{~S}$ rDNA phylogeny [27]. A comparison with $S$. agalactiae revealed less conservation of genome structure (Figure 2B), suggesting a more distant genetic relationship.

In addition to the conserved regions identified in these comparisons, discrete regions of difference were identified throughout the genome of $S$. uberis 0140J (Figure 1; Additional file 2), suggestive of diverse evolutionary origins for this component of the genome. Three discrete tracts of the sequence were identified as bacteriophage-derived islands, and a putative genomic island. When considered with additional remote CDS that are remnants of mobile genetic elements (MGEs), it was determined that MGEs constitute $1.7 \%$ of the genome. The low number of MGEs in the $S$. uberis genome is in marked contrast to other related streptococci $[28,29]$. Notably the genome does not contain any CDSs with similarity to insertion sequence (IS) elements.

Whilst the genome comparison of $S$. uberis with other related pyogenic streptococci illustrates the common evolutionary origins of these species, it is apparent from the differences in host associations and pathogenicity that they have become specialized since they diverged from their common ancestor. Insight into the functional specialisations of the $S$. uberis genome can be gleaned from a tripartite comparison with $S$. pyogenes [26] and S. zooepidemicus [25] (Figure 3). The relative compositions of the differentially shared versus unique genome components exhibit differences that illustrate niche adaptation between the species. For example, the group of CDSs shared between $S$. uberis and S. zooepidemicus encodes functions associated with central metabolism, transport and gene regulation, which are absent in the group shared between $S$. uberis and $S$. pyogenes. In comparison to $S$. uberis and $S$. zooepidemicus, $S$. pyogenes is highly nicherestricted, therefore the spectrum of substrates and stimuli it experiences is narrower. This probably explains why $S$. pyogenes does not share the broader metabolic, transport and regulatory repertoire of the other two species. The $S$. uberis-specific group contains CDSs that differentiate this species from the other two pyogenic streptococci in this three-way comparison, but also distinguish $S$. uberis from other Streptococcus species (Figure 3).

Comparison of the relative compositions of the S. uberis vs. S. zooepidemicus-S. pyogenes unique CDSs (Figure 3) and the $S$. uberis vs. other streptoccoci unique CDSs (Figure 3 ) shows similar functional makeups to each other. The functions encoded in these groups encompass a diverse range including those associated with growth (central, catabolic and energy metabolism) and host- and environmental-interactions (transport, regulators, protective responses, and cell envelope), and reflect the potential for niche adaptation by $S$. uberis. 


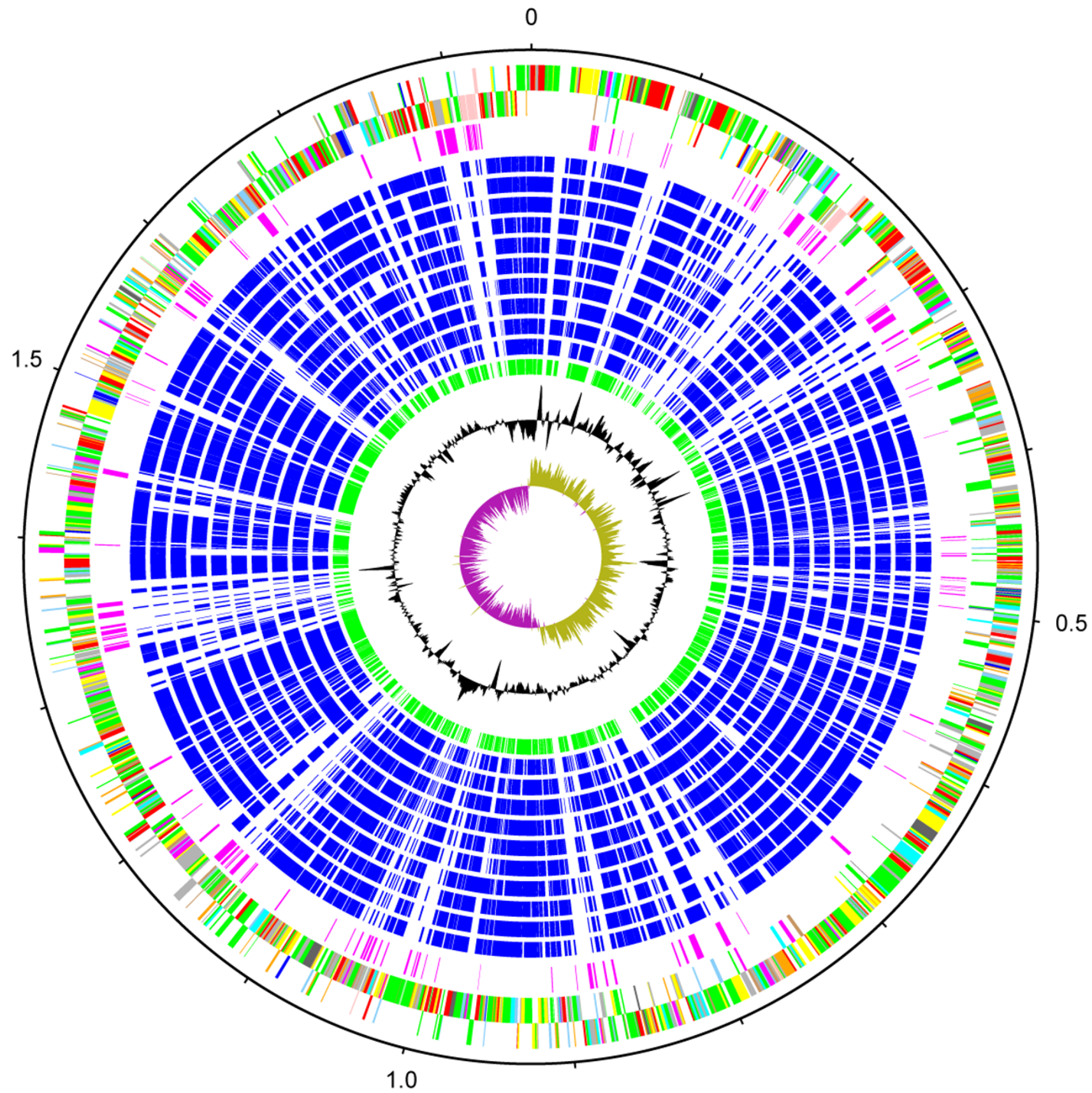

Figure I

Schematic circular diagram of the S. uberis 0 I 40J genome. Key for the circular diagram: scale (in Mb); annotated CDSs coloured according to predicted function represented on a pair of concentric circles, representing both coding strands; $S$. uberis unique CDSs, magenta; CDSs with Streptococcal ortholog matches, blue; ortholog matches shared with the Streptococcal species, S. pyogenes Manfredo, S. zooepidemicus H70, S. equi 4047, S. mutans UAI59, S. gordonii Challis CHI, S. sanguinis SK36, S. pneumoniae TIGR4, S. agalactiae NEM3 16, S. suis PI/7, S. thermophilus CNRZI 066; Lactococcus lactis subsp. lactis, green; G + C\% content plot; $G+C$ deviation plot ( $>0 \%$ olive, $<0 \%$ purple). Colour coding for CDS functions: dark blue; pathogenicity/adaptation, black; energy metabolism, red; information transfer, dark green; surface associated, cyan; degradation of large molecules, magenta; degradation of small molecules, yellow; central/intermediary metabolism, pale green; unknown, pale blue; regulators, orange; conserved hypothetical, brown; pseudogenes, pink; phage and IS elements, grey; miscellaneous. 
A

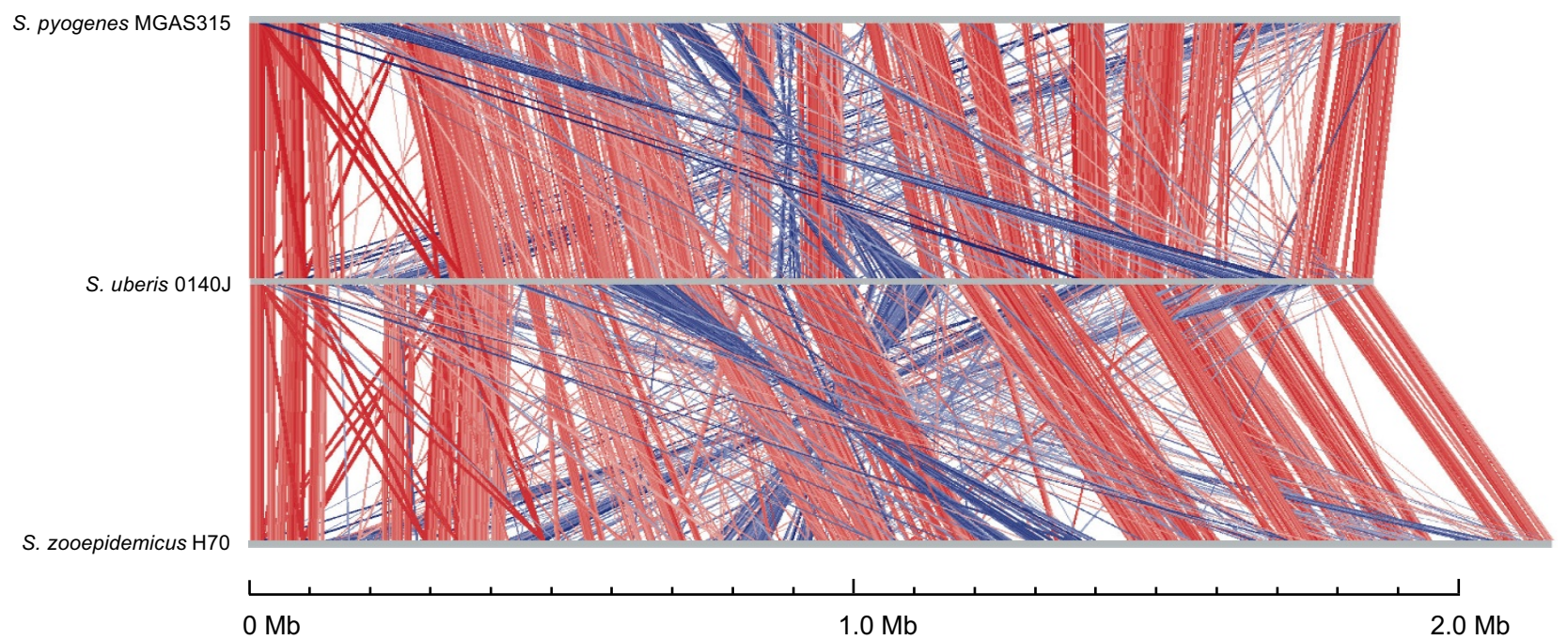

B

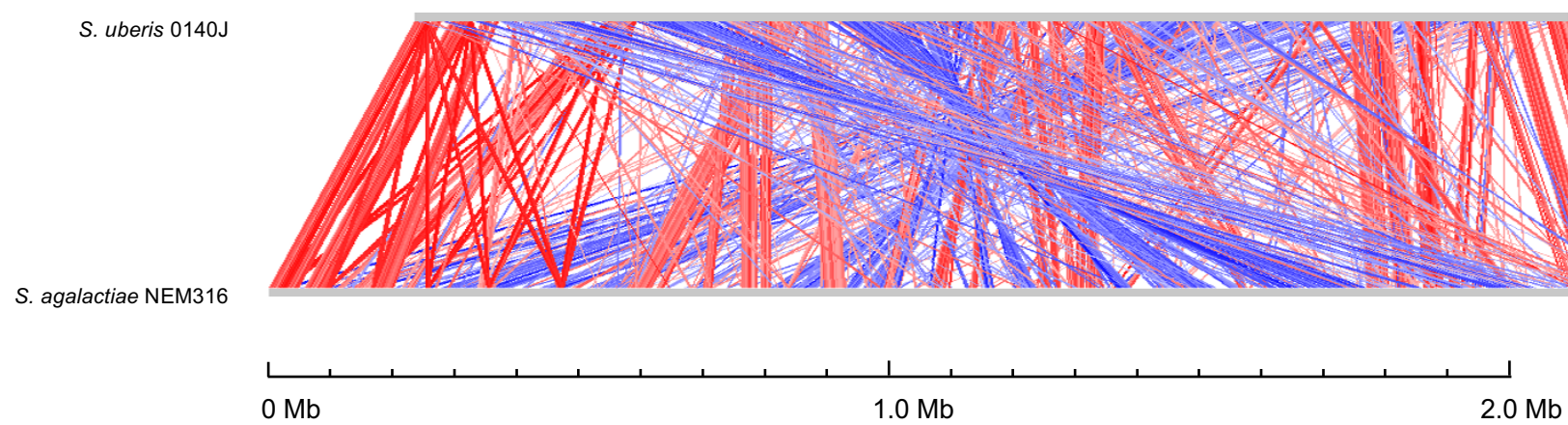

\section{Figure 2}

Genome comparison of pyogenic streptococci. Pairwise comparisons of the chromosomes of S. pyogenes MGAS3I5, S. uberis $0140 \mathrm{~J}$ and S. zooepidemicus H70 (A), and S. uberis 0I40 and agalactiae NEM3 16 (B) displayed using the Artemis Comparison Tool (ACT) [89]. The sequences have been aligned from the predicted replication origins (oriC; right). The coloured bars separating each genome (red and blue) represent similarity matches identified by reciprocal TBLASTX analysis [8I], with a score cut off of 100. Red lines link matches in the same orientation; blue lines link matches in the reverse orientation.

\section{Sugar utilization}

In comparison to other streptococci, the $S$. uberis genome contains a distinct inventory of CDSs encoding carbohydrate degradation and utilization functions. The diversity of the sugar transport and utilization apparatus in the genome provides $S$. uberis with the capacity to survive in complex host and environmental niches. In particular, $S$. uberis is well equipped to utilize microbial metabolites and products arising from the digestion of plant material found in the rumen.

S. uberis contains an expansion of glycoside hydrolase family 1 proteins [30] that catalyse the hydrolysis of glycosidic bonds between two or more carbohydrates, or between a carbohydrate and a non-carbohydrate moiety.
The S. uberis 0140J genome contains 12 members of a glycoside hydrolase family 1 (Pfam domain PF00232) in contrast to 4 in S. pyogenes Manfredo, 3 in S. equi 4047, 4 in S. zooepidemicus H70, 4 in S. suis P1/7, 6 in S. pneumoniae TIGR4, 4 in S. sanguinis SK36, 4 in S. mutans UA159, 3 in S. agalactiae NEM316, 5 in S. gordonii CH1, and 6 in Lactococcus lactis subsp. lactis IL1403. The large number of glycoside hydrolase family 1 proteins suggests that $S$. uberis has the capacity to hydrolyse a wide range of sugars. Protein sequence similarity searches (Table 1) and phylogenetic analysis (Figure 4) demonstrates the diversity of the $S$. uberis proteins. The topology of the phylogenetic tree constructed with the glycoside hydrolase family 1 proteins suggests complex evolutionary origins of the proteins (Figure 4). For example, S. uberis SUB0800 is found 


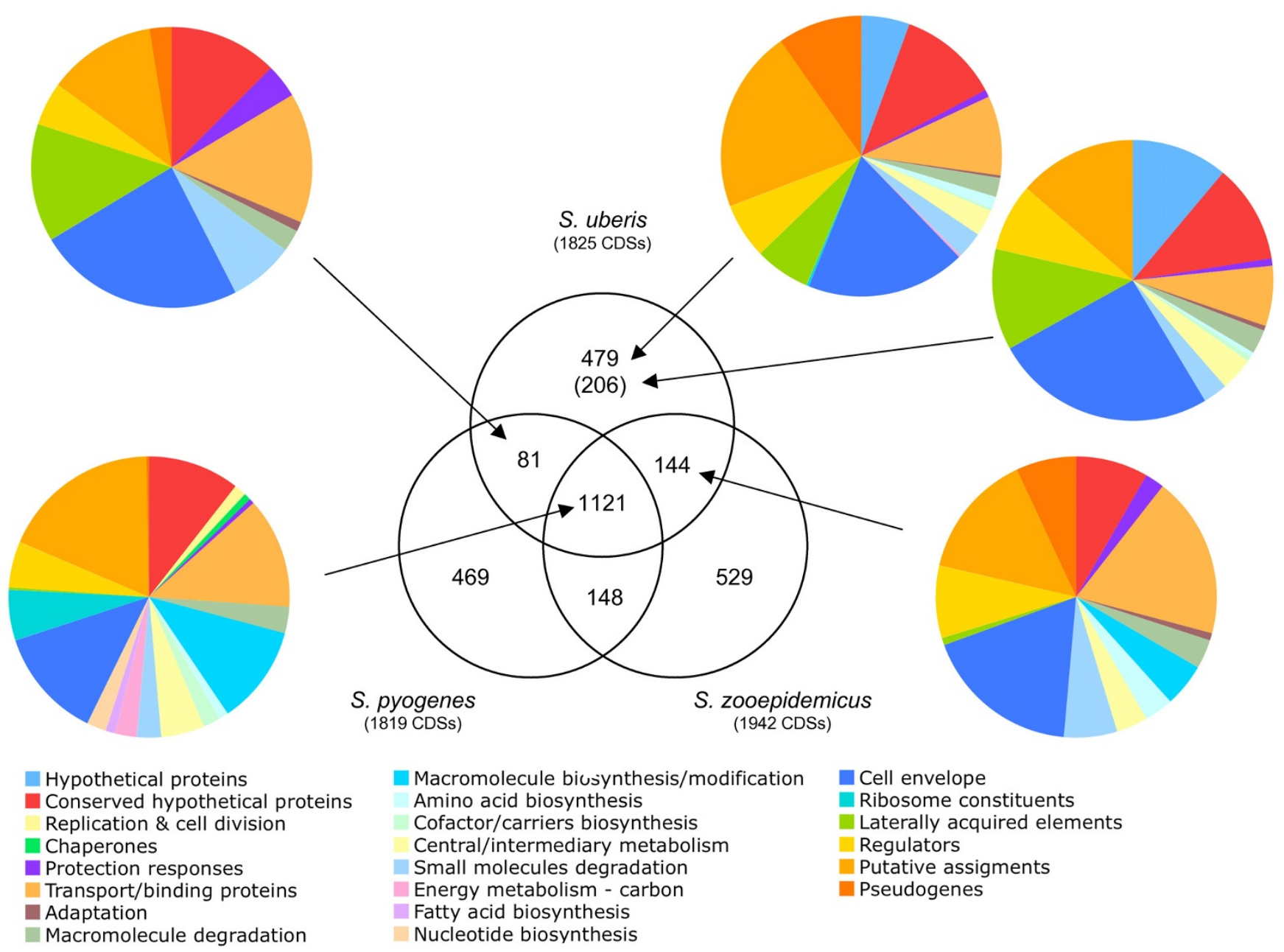

\section{Figure 3}

Distribution of orthologous CDSs in S. uberis strain 0140J, S. pyogenes strain Manfredo and S. zooepidemicus strain H70. The Venn diagram shows the number of predicted CDS unique or shared between one or more Streptococcal species. The figure in brackets indicates the number of unique CDSs that do not have ortholog matches in any of the other sequenced Streptococcus species. The relative distribution of functional groups of $S$. uberis 0I40J CDSs in the various sections is illustrated in the pie charts. Colour legend for the functional classification is below.

in a clade containing a group of orthologous proteins from different streptococcal species (S. pyogenes SpyM51607, S. zooepidemicus SZO15220, S. suis SSU0891, S. pneumoniae SpT_1100, S. sanguinis SSA_1692, S. mutans Sm_1351, S. agalactiae SaN1329, S. gordonii SGO_1512) as well as SUB1448. The branch lengths of this clade suggest that SUB0800 is more closely related to most of the other streptococcal species proteins than to SUB1448, and therefore the likely ortholog. However, comparative genomic analysis examining synteny in the regions of the $S$. uberis loci with the other streptococcal members of this clade, identified SUB1448 as the orthologous protein rather than SUB0800. Analysis of the other glycoside hydrolase family 1 proteins shows that none of them can be identified as orthologues of glycoside hydrolase family 1 proteins in closely related streptococci on the basis of synteny. It is therefore not possible to resolve the complex evolutionary origins of the glycoside hydrolase family 1 proteins in S. uberis from the limited genomic datasets currently available. It is likely that expansion in the number of members of this family in S. uberis is the result of horizontal gene transfer, possibly from outside the genus. Database searches using the family members showed that for many of the proteins, the highest levels of amino acid identity are to proteins belonging to bacteria outside the genus. The most marked example is SUB0200, where the top Fasta matches are to proteins in Gram-negative enteric bacteria (Table 1). 
Table I: Homologues of the S. uberis glycoside hydrolase family I proteins.

\begin{tabular}{|c|c|c|c|}
\hline S. uberis CDS & Protein & Organism & Identity \\
\hline \multirow[t]{3}{*}{ SUB0198 } & Q3Y0U6_ENTFC & Enterococcus faecium & $64.1 \%$ \\
\hline & Q2BFH3_9BACl & Bacillus sp. NRRL B-I49|I & $60.0 \%$ \\
\hline & Q8ES64_OCEIH & Oceanobacillus iheyensis & $60.3 \%$ \\
\hline \multirow[t]{3}{*}{ SUB0200 } & AIJLK3_YERE8 & Yersinia enterocolitica & $58.2 \%$ \\
\hline & A4WDS3_9ENTR & Enterobacter sp. 638 & $56.8 \%$ \\
\hline & Q6D574_ERWCT & Erwinia carotovora subsp. atroseptica & $57.9 \%$ \\
\hline \multirow[t]{3}{*}{ SUB0309 } & A5A692_BACLD & Bacillus licheniformis & $65.8 \%$ \\
\hline & Q65MN0_BACLD & Bacillus licheniformis & $65.8 \%$ \\
\hline & Q3XWU5_ENTFC & Enterococcus faecium & $64.2 \%$ \\
\hline \multirow[t]{3}{*}{ SUB0800 } & Q300R9_STRSU & Streptococcus suis & $99.6 \%$ \\
\hline & LACG2_STRPN & Streptococcus pneumoniae & $92.9 \%$ \\
\hline & A5M4I0_STRPN & Streptococcus pneumoniae & $92.7 \%$ \\
\hline \multirow[t]{3}{*}{ SUB0834 } & Q300V6_STRSU & Streptococcus suis & $80.9 \%$ \\
\hline & O50658_9LACO & Lactobacillus gasseri & $68.2 \%$ \\
\hline & Q04IB2_LACGA & Lactobacillus gasseri & $68.0 \%$ \\
\hline \multirow[t]{3}{*}{ SUB0837 } & Q300W6_STRSU & Streptococcus suis & $78.5 \%$ \\
\hline & Q92F20_LISIN & Listeria innocua & $61.6 \%$ \\
\hline & Q8YA94_LISMO & Listeria monocytogenes & $61.2 \%$ \\
\hline \multirow[t]{3}{*}{ SUB084I } & Q839A6_ENTFA & Enterococcus faecalis & $74.6 \%$ \\
\hline & A3CKZ2_STRSV & Streptococcus sanguinis & $73.6 \%$ \\
\hline & Q8DU50_STRMU & Streptococcus mutans & $72.2 \%$ \\
\hline \multirow[t]{3}{*}{ SUB0864 } & Q65D52_BACLD & Bacillus licheniformis & $65.8 \%$ \\
\hline & Q97TT6_CLOAB & Clostridium acetobutylicum & $62.8 \%$ \\
\hline & Q6D774_ERWCT & Erwinia carotovora subsp. atroseptica & $62.1 \%$ \\
\hline \multirow[t]{3}{*}{ SUB0905 } & Q3XXUI_ENTFC & Enterococcus faecium & $60.2 \%$ \\
\hline & Q9CFI0_LACLA & Lactococcus lactis subsp. lactis & $59.6 \%$ \\
\hline & Q3K0Z4_STRAI & Streptococcus agalactiae & $58.6 \%$ \\
\hline \multirow[t]{3}{*}{ SUBI448 } & LACG_LACLA & Lactococcus lactis subsp. lactis & $77.5 \%$ \\
\hline & Q02V89_LACLS & Lactococcus lactis subsp. cremoris & $77.3 \%$ \\
\hline & LACG_STAHJ & Staphylococcus haemolyticus & $76.8 \%$ \\
\hline \multirow[t]{3}{*}{ SUBI539 } & Q02YI9_LACLS & Lactococcus lactis subsp. cremoris & $66.7 \%$ \\
\hline & Q9CFI7_LACLA & Lactococcus lactis subsp. lactis & $63.6 \%$ \\
\hline & Q8XII5_CLOPE & Clostridium perfringens & $59.0 \%$ \\
\hline \multirow[t]{3}{*}{ SUBI579 } & A6M0G4_CLOB8 & Clostridium beijerinckii & $68.1 \%$ \\
\hline & A3UPR9_VIBSP & Vibrio splendidus & $66.2 \%$ \\
\hline & QOKKN9_STALU & Staphylococcus lugdunensis & $65.5 \%$ \\
\hline
\end{tabular}

Top 3 UniProt matches identified for each protein using FASTA.

Bacterial phosphoenolpyruvate-dependent sugar phosphotransferase systems (PTS) bind, phosphorylate, and transport sugar substrates across the bacterial cell membrane. In addition to performing sensing functions with respect to metabolite availability, components of PTS can also regulate metabolic and transcriptional processes [31].
Streptococci appear variously endowed with PTS, enabling uptake and growth on a range of carbohydrate energy sources, thereby equipping the respective species with the ability to survive and grow in a variety of ecological niches. S. pneumoniae (TIGR4) displays 21 PTS sugarspecific enzyme II complexes reflecting the utility of nutri- 


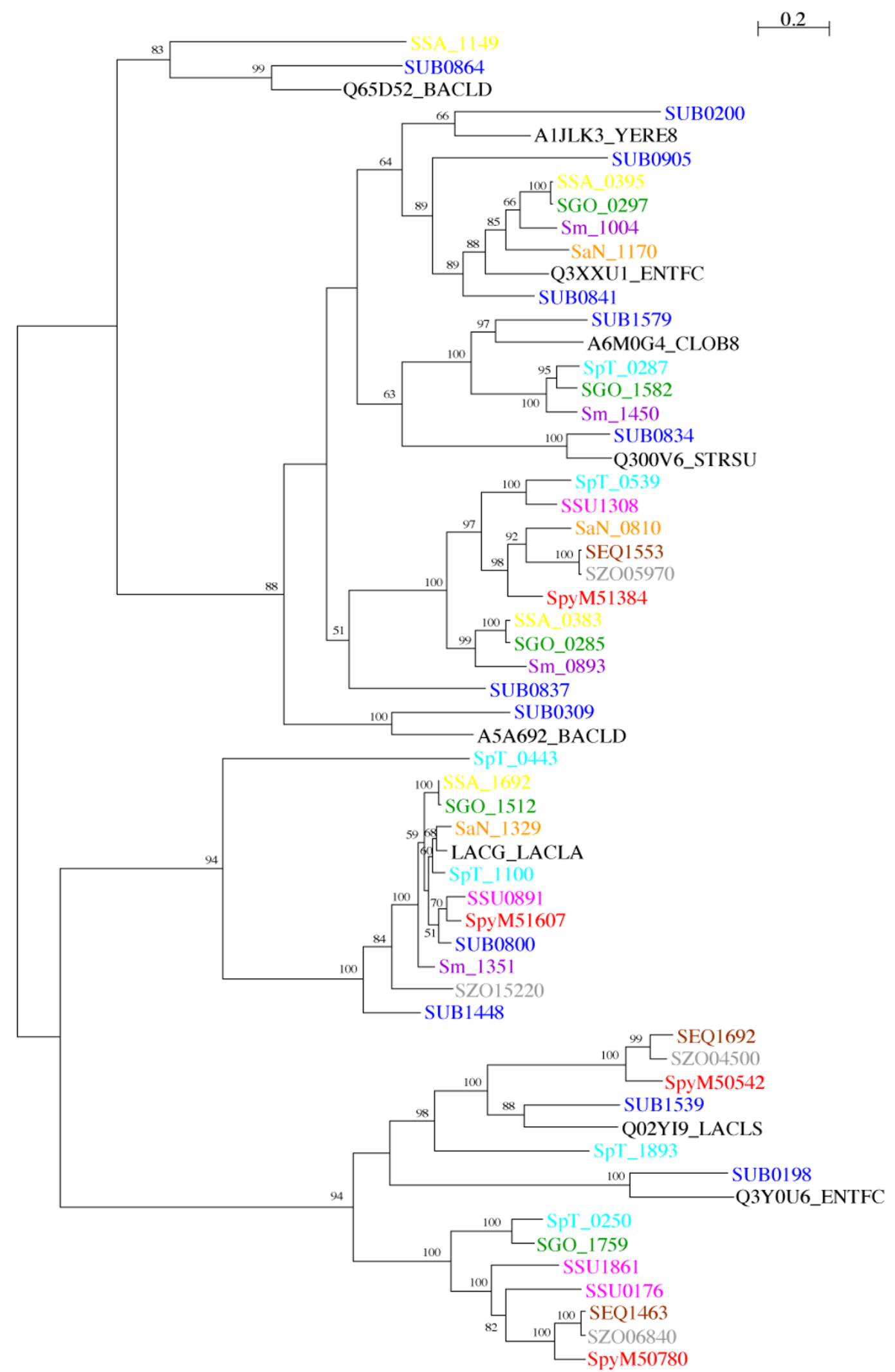

Figure 4 (see legend on next page) 
Figure 4 (see previous page)

Phylogenetic relationships of streptococcal glycoside hydrolase family I proteins. Maximum-likelihood tree of streptococcal glycoside hydrolase family I proteins from: S. uberis, dark blue; S. pyogenes Manfredo, red; S. equi 4047, brown; S. zooepidemicus H70 grey; S. suis PI/7, pink; S. pneumoniae TIGR4, S. gordonii Challis, green; light blue; S. sanguinis SK36, yellow; S. mutans UAI59, purple; S. algalactiae NEM3 I6, orange; black. Sequences of Fasta searches 'top match' hits in UniProt (Table I), outside the streptococcal genomes used above have been included, black: Q3Y0U6_ENTFC, Enterococcus faecium;

AIJLK3_YERE8, Yersinia enterocolitica; A5A692_BACLD, Bacillus licheniformis; Q300V6_STRSU, Streptococcus suis; Q65D52_BACLD, Bacillus licheniformis; Q3XXUI_ENTFC, Enterococcus faecium; LACG_LACLA, Lactococcus lactis subsp. lactis; Q02Y19_LACLS, Lactococcus lactis subsp. cremoris; A6M0G4_CLOB8, Clostridium beijerinckii. The tree was constructed with the WAG model of amino acid substitution, assuming a gamma distribution of among-site substitution rates. The numbers at the tree branches are percentage bootstrap values indicating the confidence levels, values below 50 are not shown.

tional flexibility with respect to its host niche. Similarly, some fourteen PTS were identified in another oral streptococcus, S. mutans UA159 [32]. S. uberis can call upon an array of at least fifteen PTS to satisfy the requirements of fermentative or glycolytic energy production based upon the sugars available to itwithin the bovine gut and also in mammary gland secretions. Fifteen loci were identified in the 0140J genome that contained complete PTS, with an additional five loci containing components of PTS that may represent partial or divergent systems. In comparison, the non pathogenic dairy-industry bacterium, $S$. thermophilus, was reported to have seven PTS, of which four contain pseudogenes [23].

The S. uberis 0140J genome contains a mannitol-specific PTS (SUB0288 and SUB0289) as part of an operon with a ribulose-phosphate 3-epimerase (SUB0285), 6-phospho3-hexuloisomerase (SUB0286) and a mannitol-1-phosphate 5-dehydrogenase (SUB0287). These five CDSs do not have orthologous matches in the other streptococci. The metabolic genes in this cluster encode functions for conversion of D-ribulose 5-phosphate to D-xylulose 5phosphate, isomerisation of hexulose-6-phosphate to fructose-6-phosphate and the production of D-fructose 6phosphate from D-mannitol 1-phosphate.

Concomitant with its ability to colonise the bovine gut, the lumen of the mammary gland in lactating and nonlactating animals, and its ability to survive in pasture, $S$. uberis retains numerous regulatory CDSs. Many of the regulators in the accessory genome are associated with sugar detection and metabolism. These include 6 antiterminator type regulators associated with PTS (SUB0194, SUB0530, SUB0797, SUB0829 (a pseudogene), SUB1452, SUB1704), and 4 RpiR family regulators that contain SIS phospho-sugar binding domains (SUB0170, SUB0904, SUB1541, SUB1582)

\section{Energy metabolism}

Within the CDSs unique to $S$. uberis when compared to $S$. pyogenes and $S$. zooepidemicus were two CDSs associated with energy metabolism (SUB0104 and SUB0105), that encode subunits of a cytochrome $d$ ubiquinol oxidase. These CDSs are part of an operon (SUB0102 to SUB0107) similar to the respiratory chain operon of $S$. agalactiae (menA, ndh, cydA, cydB, cydC, and cydD) [33]. This respiratory chain is incomplete in S. uberis, as it is in S. agalactiae, as the genome does not encode the biosynthetic pathways for quinone, required for electron transfer, and haem, a cytochrome oxidase cofactor. However respiration in $S$. agalactiae can be stimulated under aerobic conditions if exogenous haem and quinone are supplied [33]. The presence of two distinct metabolic routes for energy production, fermentation and respiration, bestows $S$. uberis with a metabolic versatility that may promote survival in the diverse niches it occupies. In vitro experiments with $S$. agalactiae have shown a survival advantage for cells grown under respiratory conditions as opposed to under fermentation conditions [33]. Mutants of cytochrome $d$ ubiquinol oxidase demonstrated lower levels of growth in blood under aerobic conditions, and also had reduced virulence in a neonatal rat sepsis model [33]. The ability to respire aerobically may be important for the spread and dissemination of $S$. uberis, although the requirement for exogenous haem and quinone suggest that this is strongly linked to environmental conditions dictated by the host or niche microbiota.

A recent study showed that quinones produced by Lactococcus lactis cross-feed S. agalactiae and activate respiration when the two organism were co-cultured [34]. Given the complexity of the microbial ecosystems in which $S$. uberis resides, it is not unreasonable to hypothesize that heme and quinone would be available permitting reconstitution of the respiratory chain. Cross-feeding of these key respiratory molecules by resident bacteria in the lower gastrointestinal tract may promote the fecal shedding of $S$. uberis. Whilst the anaerobic conditions of the gut may preclude respiration in this environment, once outside the gut it is possible that a shift in energy metabolism may promote growth and/or survival of $S$. uberis in fecal matter. 


\section{Protective responses and environmental survival}

The $S$. uberis genome encodes a polyphosphate kinase (SUB0262), a phosphate metabolism enzyme absent in other streptococci. This enzyme catalyzes the reversible transfer of the terminal phosphate of ATP to form a longchain polyphosphate (polyP). The accumulation of polyP within E. coli cells has been shown to be a response to nutritional and osmotic stresses [35], and polyP has been demonstrated to be essential for long-term survival of Shigella and Salmonella spp. [36]. In Vibrio cholerae, polyphosphate stores enhance the ability of to overcome environmental stresses in a low-phosphate environment [37]. The presence of this enzyme in S. uberis suggests that this organism is equipped to tolerate comparatively low phosphate-availability environments such as those that might be encountered in faeces and pasture. Recent studies have also demonstrated a role for polyphosphate kinase in the pathogenicity of Salmonella and Camylobacter species [38,39].

Quaternary ammonium compound (QAC) disinfectants are used routinely as part of the everyday milking procedure in dairies as a hygiene measure. The S. uberis 0140J genome contains a putative quaternary ammonium compound-resistance protein (SUB0162) that has 41\% identity with QACH_STASA the quaternary ammonium compound-resistance protein QacH from Staphylococcus saprophyticus plasmid pST2H6 [40]. Downstream of this CDS there are two other CDSs that make a putative operon: SUB0163, binding-protein-dependent transport system membrane protein; and SUB0164, a fusion protein pseudogene. This latter CDS consists of an N-terminus similar to $\mathrm{ABC}$ transporter permease proteins and a Cterminus similar to fibronectin binding protein, containing an LPXTG motif. It is likely that this CDS arose due to a deletion event that resulted in the fusion of these functionally distinct domains. The three CDSs make up an operon, however it is not apparent what effect, if any, the mutation in SUB0164 will have upon resistance to QACs.

A distinct feature of the S. uberis genome that has emerged from the comparative genomic analysis with other streptococcal species is that there is an abundance of bacteriocins and associated processing, transport and immunity proteins. With the exception of the mammary gland, the niches that $S$. uberis colonize are highly competitive, and are populated by a diverse microbiota. Bacteriocins are proteinaceous antibiotics produced by bacteria that typically kill other bacteria of the same or closely related species. The production of bacteriocins by $S$. uberis is thought to provide a competitive edge and promote colonization.

Uberolysin is a novel cyclic bacteriocin produced by $S$. uberis (SUB0032 to SUB0036) [41]. It has been reported that some strains of $S$. uberis produce nisin U, a lantibi- otic, which is similar to nisin A produced by Lactococcus lactis [42]. The $16 \mathrm{~kb}$ nisin $\mathrm{U}$ biosynthesis and resistance locus is absent in S. uberis 0140J. The genome also contains a locus (SUB0502 to SUB0516) that encodes an array of bacteriocin structural proteins and immunity proteins. In total there are six CDSs encoding putative bacteriocin proteins (SUB0502, SUB0503, SUB0505, SUB0506, SUB0509 and SUB0512). The presence of multiple structural and immunity CDSs suggests a degree of redundancy. Several of the CDSs at this locus are pseudogenes (two of which are bacteriocin structural CDSs), suggesting that recent mutations have altered the bacteriocin expression repertoire of strain 0140J, and that there may be have been selection for the differential expression of CDSs at this locus. There is evidence of allelic variation at this locus in other strains of S. uberis; S. uberis strain E, contains a gene encoding (UbaA), a 5.3-kDa class IIa (pediocin-like) bacteriocin at this locus [43], whereas strain 0140J lacks $u b a A$, but contains divergent bacteriocin CDSs. Notably the ubericin A putative immunity protein UbaI is conserved in both strains (SUB0516; 98\% identical at the amino acid level).

$3.4 \%$ of the S. uberis $0140 \mathrm{~J}$ genome encodes pseudogenes (Additional file 1). Comparable levels are observed in the genomes of S. suis (strain P1/7) (unpublished data) and $S$. pyogenes (Manfredo) [26]; other Gram positive pathogens of similarly low GC content such as Staphylococcus aureus have a pseudogene content of between 0.8 and $2.5 \%$ $[44,45]$. These figures are significantly below the $10 \%$ figure reported for two S. thermophilus strains (CNRZ1066, LMG18311) for which disruption due to numerous mobile elements and an ongoing depletion of superfluous carbon utilisation CDS were deemed contributory factors [23]. Statistical analysis suggests that pseudogenes are over-represented in genomes of bacteria experiencing either new environmental niches or those becoming equipped to live in a diversity of habitats [45]. Although the relative level of pseudogenes is not high, it is a point of debate that the high representation of regulators and surface-associated proteins within this (Additional file 1), is suggestive of an organism engaged in the process of adapting to the challenge of successfully colonising the bovine gut and the mammary gland.

\section{Virulence}

The majority of virulence factors in $S$. uberis genome are found in the variable component of the genome (Figure 3 ) although some are also found in the core gene set, for example fibronectin-binding protein (SUB1111) [46], hemolysin-like protein (SUB1273), and a C5A peptidaselike precursor (SUB1154). Overall there was a lack of what might be considered classical virulence determinants when compared with S. pyogenes. For example, genes associated with the production and surface anchoring of $\mathrm{M}$ 
protein in S. pyogenes are absent from S. uberis 0140J. Orthologs of emm and mga (a transcriptional activator of emm) were not present and none of the CDSs identified previously as putative sortase substrates [47] suggested Mlike properties. Other structural proteins that utilise sortase-mediated surface-anchoring such as pilin are also absent from the genome. As a pathogen, S. zooepidemicus has been less intensively studied, therefore the paucity of virulence determinants in this species, and consequently identified by comparison with $S$. uberis, reflects this. Detailed analysis of the $S$. uberis genome however identified several CDSs that have potential roles in virulence.

Pathogenic bacteria display an assortment of cell surfaceassociated proteins that can interact with host cells and tissues and contribute to the pathogenicity of the organism. Bacteria utilise a number of different mechanisms to attach proteins to the cell wall, one of which is through covalent linkage initiated by sortase enzymes. Up to 5 different classes of sortase enzymes have been identified in Gram positive bacteria $[48,49]$ with one gene identified in the $S$. uberis genome, srtA (SUB0881). Anchoring of protein A of Staphylococcus aureus via SrtA has been well characterised [50]. Proteins that are destined for anchoring by sortase typically have N-terminal signal sequences for protein secretion and a cell wall sorting signal consisting of an LPXTG, or occasionally an LPX(A/S)G, anchoring motif followed by a C-terminal hydrophobic region with a positively charged tail of amino acids [51].

Seven genes were identified that contained LPXTG sorting motifs; SUB0145, SUB0164, SUB0348, SUB0888, SUB1095, SUB1730 and SUB1739 and a further two (SUB0207 and SUB0241) containing LPXAG motifs. However, two of these, SUB0164 and SUB1739, are highly likely to be pseudogenes and thus unlikely to be translated into fully functional, anchored proteins. A number of these genes have similarity with previously characterised streptococcal proteins; SUB0145, encodes a lactoferrin binding protein (Lbp) [52] which displays homology to fibrinogen-binding/M-like proteins, and SUB1095 shows similarity to an array of SclB collagen-like surface proteins of $S$. pyogenes [53]. Four additional CDSs, SUB0135, a fructan beta-fructosidase precursor and SUB0764, a surface-anchored 5'-nucleotidase (pseudogene), SUB0826, a subtilase family protein, and SUB1154, a C5A peptidase-like precursor, are also putative sortaseprocessed surface-anchored CDSs, containing the variant motifs LPXTS, LPXTS, LPXTR and LPXTV respectively.

A typical response to intra-mammary infection by the host is an increase in lactoferrin, an iron-binding glycoprotein that acts to restrict the availability of iron for bacterial growth $[54,55]$. Streptococci are considered to be resistant to the antibacterial effects of lactoferrin, most likely due to the low requirement for iron for growth [56]. However, the ability to sequester iron from host glycoproteins is considered to be essential for bacterial pathogenesis and bacterial iron-binding proteins have been investigated as potential virulence factors. Within the $S$. uberis genome both Lbp (SUB0145) and S. uberis adhesion molecule or SUAM proteins (SUB1635) have been investigated as potential iron-binding proteins $[52,57,58]$. The SUAM protein has an affinity for lactoferrin and is involved in the adherence of $S$. uberis to a bovine mammary epithelial cell line in vitro and therefore may be important during the initial stages of infection and colonisation [57,59]. Adherence experiments using $l b p$ mutants however, suggest that Lbp is not required for attachment of $S$. uberis to host epithelial cells and it is also not essential for growth in iron-limiting conditions. The predicted protein sequence of Lbp is weakly similar ( $28 \%$ identity) to the streptococcal $M$ proteins, and the genome also contains a partial CDS (SUB0144) with homology to VirR12 and Mry which are positive regulators of $M$ protein genes in group A streptococci [52]. The M protein and C5a peptidase are well characterised virulence determinants of $S$. pyogenes which act on different arms of the complement pathway, inhibiting bacterial opsonisation and inflammation and also phagocyte recruitment to the site of infection $[60,61]$. In $S$. pyogenes, these virulence determinants contain LPXTG motifs.

The hyaluronic acid capsule of $S$. pyogenes has been implicated in the pathogenesis of invasive GAS infections [62] and is essential for the maturation of GAS biofilms [63]. Strains of $S$. uberis isolated from cases of bovine mastitis display variable amounts of hyaluronic acid capsule. The complement and arrangement of hyaluronic acid biosynthetic genes in $S$. uberis differs from the has $A B C$ arrangement common to GAS [64]. The has operon, consisting of hasA (SUB1697) encoding hyaluronan synthase and has $B$ (SUB1696) encoding UDP-glucose dehydrogenase, is similarly arranged to that in GAS. However a has $C$ homologue (SUB1691), encoding UDP-glucose pyrophosphorylase, was identified downstream of has $B$ in the reverse orientation and separated from it by some $3 \mathrm{~kb}$ containing CDSs apparently unrelated to capsule biosynthesis. The hasA gene product is essential for capsule production in both S. pyogenes and S. uberis $[64,65]$. However, capsule production in GAS is dependent only upon functional has $A$ and has $B$, but not hasC [66]. The implication of functional redundancy for UDP-glucose pyrophosphorylase activity in GAS was further supported by the identification of additional homologous CDS in multiple GAS genomes. In contrast, lesions in isogenic acapsular $S$. uberis $0140 \mathrm{~J}$ mutants were confined to the has $A$ and has $C$ open reading frames suggesting no redundancy existed for these enzymes [64]. Correspondingly, a homologue (SUB1027) of the $S$. uberis hasB gene product (SUB1696) 
was identified that might account for the apparent inability to isolate a hasB mutant using phenotypic screening methods [64].

The lesions identified in non-mucoid mutants of $S$. uberis 0140J are distributed between genetic loci likely to be involved in biosynthesis and also regulation. Three independent acapsular insertion mutants mapped within a putative GntR-like transcriptional regulator SUB0978 (unpublished data) suggestive of a role in upregulation of capsule precursors. The exploitation of an isogenic mutant lacking functional HasA demonstrated that the hyaluronic acid capsule of $S$. uberis plays little role in the early stages of infection of the lactating bovine mammary gland, and resistance to phagocytosis was ascribed to an undefined component unconnected with the capsular phenotype [67].

Activation of host plasminogen to plasmin, a potent serine protease that can bind to the surface of numerous bacterial pathogens, is a mechanism thought likely to augment a variety of streptococcal infections. Many of the streptococci within the pyogenic cluster express one of a variety of plasminogen activators that associate with the respective host's plasminogen to form an activation complex capable of recruiting and activating further substrate plasminogen to plasmin. The considerable sequence diversity, various substrate specificities and origins of the streptococcal plasminogen activators remain subjects of considerable research interest. The overwhelming majority of S. uberis isolates encode PauA (SUB1785) located between the hexA and hexB genes which display homology to the highly prevalent mutS and mutL DNA mismatch repair genes [68]. A single $S$. uberis isolate lacking pauA encoded an alternative novel plasminogen activator (PauB) [69] also at the hexAB locus.

An alternative locus in all human isolates of group A, C and G streptococci is populated by streptokinase (SK) [70]. The genes flanking SK did not appear to encode related functions, being an ATP-binding cassette protein and a leucine rich protein lying upstream of ska, while genes encoding a homologue of the E. coli relA and spoT genes which moderate levels of guanosine $5^{\prime}, 3^{\prime}$ polyphosphate during nutritional stress lie downstream [71]. The corresponding SK locus in S. uberis 0140J is devoid of plasminogen activator CDS.

Some clues concerning the origins of the diversity of plasminogen activator sequences and the variety of genomic loci within which they appear in pyogenic streptococci are drawn from the observation that in at least three $S$. pyogenes serotypes (M1, M5 and M6) $[26,72,73]$, prophage are located between the hexA and hexB orthologues with the phage attachment site for SF370.4 being mapped to overlie the start of the mutL coding sequence [72]. Further evidence of the role that phage play in generating variation at this locus is found in the CDS upstream of pauA, which encodes a putative exported protein (SUB1784), and is similar to proteins from $L$. lactis prophage.

Hyaluronidase, has long been considered to be a streptococcal virulence determinant due to its ability to break down the hyaluronic acid component of connective tissue and thereby facilitate spread of the pathogen, but there is little experimental evidence to support this assertion. Hyaluronic acid capsule produced by some streptococci, including S. uberis, is somewhat paradoxically also a substrate for hyaluronidase. Recently, it has been suggested that hyaluronidase facilitates the spread of high molecular weight compounds rather than bacterial cells per se [74] and also augments the effects of other streptococcal virulence factors such as pneumolysin [75]. In GAS, hylA encodes a large secreted hyaluronidase. In addition, numerous 'phage-associated' hyaluronidases $(h y l P)$ are evident. Neither GAS hylA or hylP homologues were present in the $S$. uberis $0140 \mathrm{~J}$ sequence. Interestingly the GBS2603 V/R locus encoding a chromosomal hyaluronidase (SAG1197) abuts $m u t X$ with the equivalent locus in GAS Manfredo being the prophage phiman.3, encoding one of a number of M5 phage hyaluronidase CDS. It appears highly likely that the hyaluronidase complement of streptococci has been and continues to be influenced by bacteriophage.

The CAMP factor [76], a further putative virulence factor homologous to the Fc-binding and haemolytic $c f b$ gene product in S. agalactiae is absent from numerous strains of S. uberis [77]. A CDS encoding CAMP factor was not identified in S. uberis 0140J or within 8 of a panel of 9 further pathogenic $S$. uberis strains tested (unpublished data) and can therefore be considered as non-essential to the infection of the bovine mammary gland.

\section{Conclusion}

Analysis of the whole genome sequence of the bovine mastitis associated S. uberis strain 0140J has identified a relative paucity of classical streptococcal virulence determinants. In contrast, a plethora of metabolic options appear available to the bacterium. These endow it with the potential to flourish in a variety of nutritionally constrained habitats including the lumen of the mammary gland and the bovine gut. There are evidently survival advantages for an organism able to occupy multiple environmental niches. Critical to this aspect is nutritional flexibility, and the ability to derive carbon and energy by a variety of means. The genome provides compelling evidence supporting this proposal. Furthermore, S. uberis also appears equipped to cope with stresses likely to be encountered when shifting from one environmental niche 
to another for example through the use of polyphosphate kinase (SUB0262). We present here evidence that suggests $S$. uberis should no longer be regarded merely as a pathogen of the bovine mammary gland, but equally as a commensal microorganism of a wider bovine-based environment. Within this context, it will be of great interest to determine the current distribution of such features within the species and also whether $S$. uberis continues to refine or augment its genetic load along such lines in the future.

\section{Methods \\ Strain}

Streptococcus uberis 0140J was isolated from milk obtained from a clinical case of bovine mastitis in 1972 within the UK. The strain S. uberis 0140J has been deposited under ATCC ${ }^{\circledR}$ Number BAA-854.

\section{Preparation of genomic DNA}

Genomic DNA was prepared from S. uberis using a modification of the method of Hill and Leigh [78]. Todd Hewitt broth $(400 \mathrm{ml})$ was inoculated with a single colony of $S$. uberis 0140J picked from solid media and incubated for $18 \mathrm{~h}$ at $37^{\circ} \mathrm{C}$. Bacterial cells were harvested, washed with $180 \mathrm{ml}$ of Tris, $5 \mathrm{mM}$ EDTA (pH 7.8) and the cell walls disrupted by incubation at $37^{\circ} \mathrm{C}$ for 30 mins in $50 \mathrm{ml}$ of the same buffer, containing $30 \mathrm{u} / \mathrm{ml}$ mutanolysin and 10 $\mathrm{mg} / \mathrm{ml}$ lysozyme. Cells were lysed by addition of $2.67 \mathrm{ml}$ SDS (20\% w/v in $50 \mathrm{mM}$ Tris, $20 \mathrm{mM}$ EDTA pH 7.8), 0.4 $\mathrm{ml}$ Proteinase $\mathrm{K}(20 \mathrm{mg} / \mathrm{ml})$ and incubation at $37^{\circ} \mathrm{C}$ for 1 h. Saturated $\mathrm{NaCl}(26.7 \mathrm{ml})$ was added and after $5 \mathrm{~min}$. cell wall material was removed by centrifugation for 20 mins at $16,000 \times g$. The supernatent was extracted twice with an equal volume of Tris-equilibrated ( $\mathrm{pH} 8.0)$ phenol:chloroform:isoamyl alcohol (25:24:1). RNAase A was added to $20 \mu \mathrm{g} / \mathrm{ml}$ and the mixture incubated for $30 \mathrm{mins}$ at $37^{\circ} \mathrm{C}$. Genomic DNA was precipitated by addition of 2 volumes of ice-cold ethanol and incubation at $4{ }^{\circ} \mathrm{C}$ for 2 $\mathrm{h}$ prior to centrifugation at $16,000 \times g$ for 15 mins. The pelleted DNA was washed with cold $70 \%$ ethanol and airdried prior to resuspension at $4{ }^{\circ} \mathrm{C}$ in $2.5 \mathrm{ml}$ of TE buffer.

\section{Whole genome sequencing}

Sequence data were obtained from end sequences (giving approximately $8 \times$ coverage) derived from $\mathrm{M} 13$ and pUC18 genomic shotgun libraries (with insert sizes of 1.4 to $2 \mathrm{~kb}$ and 2.8 to $3.3 \mathrm{~kb}$ respectively) using dye terminator chemistry on ABI3700 automated sequencers. End sequences from a large insert BAC library (pBACe3.6, 12 to $48 \mathrm{~kb}$ insert size) and Fosmid library (pEpiFos, 30-40 $\mathrm{kb}$ ) were used as a scaffold. All identified repeats were bridged by read-pairs or end-sequenced polymerase chain reaction (PCR) products. The sequence was assembled and finished as described previously [79].
The sequence was annotated using Artemis software [79]. Initial coding sequence (CDS) predictions were performed using Orpheus [80], Glimmer2 [81], and EasyGene [82] software. These predictions were amalgamated, and codon usage, positional base preference methods and comparisons to the non-redundant protein databases using BLAST [83] and FASTA [84] software were used to refine the predictions. The entire DNA sequence was also compared in all six reading frames against UniProt, using BLASTX [83] to identify any possible coding sequences previously missed. Protein motifs were identified using Pfam [85] and Prosite [86], transmembrane domains were identified with TMHMM [87], and signal sequences were identified with SignalP version 2.0 [88]. rRNAs were identified using BLASTN [83] alignment to defined rRNAs from the EMBL nucleotide database; tRNAs were identified using tRNAscan-SE [89]; non-coding RNA (ncRNA) were identified using Rfam [90]. The sequence and annotation of the Streptococcus uberis 0140J genome has been deposited in the EMBL database under accession number AM946015.

\section{Comparative genomics}

Comparison of the genome sequences was facilitated by using the Artemis Comparison Tool (ACT) [91] which enabled the visualization of BLASTN and TBLASTX comparisons [83] between the genomes. Orthologous proteins were identified as reciprocal best matches using FASTA [84] with subsequent manual curation. Orthology inferred from positional information was investigated using ACT. Pseudogenes had one or more mutations that would prevent correct translation; each of the inactivating mutations was subsequently checked against the original sequencing data.

Streptococcus sequences used for comparative genomic analysis were: $S$. pyogenes Manfredo (accession number AM295007) [26], S. pyogenes MGAS315 (accession number AE014074) [92], S. equi 4047 http:// www.sanger.ac.uk/Projects/S equi/[25], S. zooepidemicus H70 http://www.sanger.ac.uk/Projects/S zooepidemicus/ [25], S. thermophilus CNRZ1066 (accession number CP000024) [23], S. suis P1/7 http://www.sanger.ac.uk/ Projects/S_suis/[93], S. pneumoniae TIGR4 (accession number AE005672) [94], S. sanguinis SK36 (accession number $\underline{\mathrm{CP000387}}$ ) [95], S. mutans UA159 (accession number $\underline{\mathrm{AE} 014133}$ ) [32], S. agalactiae NEM316 (accession number AL732656) [96], and S. gordonii str. Challis substr. CH1 (accession number CP000725) [97], and Lactococcus lactis subsp. lactis IL1403 (accession number AE005176) [98].

\section{Phylogentic analysis}

Unrooted maximum likelihood trees were built using PhyML [99,100] and drawn using NJplot [101]. 
Sequences were aligned using ClustalX (Version 1.82) [102]

\section{Abbreviations}

ACT: Artemis Comparison Tool; ARF: acute rheumatic fever; CDS: coding sequences; GAS: group A Streptococcus; IS: insertion sequence; Lbp: Lactoferrin-binding protein; PolyP: polyphosphate; PTS: phosphotransferase system; QAC: quaternary ammonium compound; SK: streptokinase; TSS: toxic shock syndrome.

\section{Authors' contributions}

PNW and SAE drafted the manuscript, MTGH annotated the genome and drafted the manuscript, JL conceived of the study, participated in the study design, and drafted the manuscript, MAQ and JW carried out the genomic library preparation, $\mathrm{NL}, \mathrm{ABi}, \mathrm{ABa}, \mathrm{LC}$, carried out the genome finishing and closure, DM and AW conceived of the study, participated in the study design and undertook critical review of the manuscript, TRF, MK, CD and NC conceived of the study, participated in the study design, $\mathrm{BH}, \mathrm{BGB}, \mathrm{JP}$ and SDB participated in the study design, managed the project and undertook critical review of the manuscript. All authors have read and approved the final manuscript.

\section{Additional material}

\section{Additional File 1}

Table Pseudogenes S1: and partial genes in the S. uberis 0140J genome. Table S1: Table containing a list of the predicted products, systematic IDs, and putative mutations associated with pseudogenes and partial genes in the S. uberis 0140J genome.

Click here for file

[http://www.biomedcentral.com/content/supplementary/14712164-10-54-S1.pdf]

\section{Additional File 2}

Unique CDSs compared to other sequenced streptococci. Table containing a list of the systematic IDs and predicted products for CDSs in the S. uberis $0140 J$ genome that do not have reciprocal Fasta matches in $\mathrm{S}$. pyogenes Manfredo, S. equi 4047, S. zooepidemicus H70, S. thermophilus CNRZ1066, S. suis P1/7, S. pneumoniae TIGR4, S. sanguinis SK36, S. mutans UA159, S. agalactiae NEM316, and S. gordonii str. Challis substr. $\mathrm{CH} 1$.

Click here for file

[http://www.biomedcentral.com/content/supplementary/14712164-10-54-S2.pdf]

\section{Acknowledgements}

This work was supported by the Wellcome Trust. We would like to acknowledge the support of the Wellcome Trust Sanger Institute's Pathogen Production Group for shotgun sequencing and finishing.

\section{References}

I. Gottschalk M, Segura M, Xu J: Streptococcus suis infections in humans: the Chinese experience and the situation in North America. Anim Health Res Rev 2007, 8:29-45.

2. Bisharat N, Crook DW, Leigh J, Harding RM, Ward PN, Coffey TJ, Maiden MC, Peto T, Jones N: Hyperinvasive neonatal group $B$ streptococcus has arisen from a bovine ancestor. J Clin Microbiol 2004, 42:216I-2167.

3. Bentley RW, Leigh JA, Collins MD: Intrageneric structure of Streptococcus based on comparative analysis of small-subunit rRNA sequences. Int J Syst Bacteriol I99I, 41:487-494.

4. Compton CW, Heuer C, Parker K, McDougall S: Epidemiology of mastitis in pasture-grazed peripartum dairy heifers and its effects on productivity. J Dairy Sci 2007, 90:4I 57-4I70.

5. Olde Riekerink RG, Barkema HW, Kelton DF, Scholl DT: Incidence rate of clinical mastitis on Canadian dairy farms. J Dairy Sci 2008, 9 I: I366-I377.

6. Sorensen LP, Guldbrandtsen B, Thomasen JR, Lund MS: Pathogenspecific effects of quantitative trait loci affecting clinical mastitis and somatic cell count in Danish Holstein cattle. J Dairy Sci 2008, 91 1:2493-2500.

7. Bradley AJ, Leach KA, Breen JE, Green LE, Green MJ: Survey of the incidence and aetiology of mastitis on dairy farms in England and Wales. Vet Rec 2007, I 60:253-257.

8. Leigh JA: Streptococcus uberis: a permanent barrier to the control of bovine mastitis? Vet J 1999, I 57:225-238.

9. Bramley AJ: Sources of Streptococcus uberis in the dairy herd. I. Isolation from bovine faeces and from straw bedding of cattle. J Dairy Res 1982, 49:369-373.

10. Kruze J, Bramley AJ: Sources of Streptococcus uberis in the dairy herd. II. Evidence of colonization of the bovine intestine by Str. uberis. J Dairy Res 1982, 49:375-379.

II. Lopez-Benavides MG, Williamson JH, Pullinger GD, Lacy-Hulbert SJ, Cursons RT, Leigh JA: Field observations on the variation of Streptococcus uberis populations in a pasture-based dairy farm. J Dairy Sci 2007, 90:5558-5566.

12. Hill AW: Pathogenicity of two strains of Streptococcus uberis infused into lactating and non-lactating bovine mammary glands. Res Vet Sci 1988, 45:400-404.

13. Sordillo LM, Shafer-Weaver K, DeRosa D: Immunobiology of the mammary gland. J Dairy Sci 1997, 80: 185 I- I865.

14. Coffey TJ, Pullinger GD, Urwin R, Jolley KA, Wilson SM, Maiden MC, Leigh JA: First insights into the evolution of Streptococcus uberis: a multilocus sequence typing scheme that enables investigation of its population biology. Appl Environ Microbiol 2006, 72:1420-|428.

15. Leigh JA, Ward PN, Field TR: The exploitation of the genome in the search for determinants of virulence in Streptococcus uberis. Vet Immunol Immunopathol 2004, I 00: I45- I 49.

16. Ferretti JJ, McShan WM, Ajdic D, Savic DJ, Savic G, Lyon K, Primeaux C, Sezate S, Suvorov AN, Kenton S, Lai HS, Lin SP, Qian Y, Jia HG, Najar FZ, Ren Q, Zhu H, Song L, White J, Yuan X, Clifton SW, Roe $B A$, McLaughlin R: Complete genome sequence of an MI strain of Streptococcus pyogenes. Proc Natl Acad Sci USA 200I, 98:4658-4663.

17. Tettelin H, Masignani V, Cieslewicz MJ, Eisen JA, Peterson S, Wessels MR, Paulsen IT, Nelson KE, Margarit I, Read TD, Madoff LC, Wolf AM, Beanan MJ, Brinkac LM, Daugherty SC, DeBoy RT, Durkin AS, Kolonay JF, Madupu R, Lewis MR, Radune D, Fedorova NB, Scanlan D, Khouri H, Mulligan S, Carty HA, Cline RT, Van Aken SE, Gill J, Scarselli M, Mora M, lacobini ET, Brettoni C, Galli G, Mariani M, Vegni F, Maione D, Rinaudo D, Rappuoli R, Telford JL, Kasper DL, Grandi G, Fraser CM: Complete genome sequence and comparative genomic analysis of an emerging human pathogen, serotype V Streptococcus agalactiae. Proc Natl Acad Sci USA 2002, 99: I2391-12396.

18. Shen K, Gladitz J, Antalis P, Dice B, Janto B, Keefe R, Hayes J, Ahmed A, Dopico R, Ehrlich N, Jocz J, Kropp L, Yu S, Nistico L, Greenberg DP, Barbadora K, Preston RA, Post JC, Ehrlich GD, Hu FZ: Characterization, distribution, and expression of novel genes among eight clinical isolates of Streptococcus pneumoniae. Infect Immun 2006, 74:32I-330.

19. Hiller NL, Janto B, Hogg JS, Boissy R, Yu S, Powell E, Keefe R, Ehrlich NE, Shen K, Hayes J, Barbadora K, Klimke W, Dernovoy D, Tatusova T, Parkhill J, Bentley SD, Post JC, Ehrlich GD, Hu FZ: Comparative genomic analyses of seventeen Streptococcus pneumoniae 
strains: insights into the pneumococcal supragenome. J Bacteriol 2007, 189:8186-8195.

20. Ferretti JJ, Ajdic D, McShan WM: Comparative genomics of streptococcal species. Indian J Med Res 2004, I I 9(Suppl): I-6.

21. Marri PR, Hao W, Golding GB: Gene gain and gene loss in streptococcus: is it driven by habitat? Mol Biol Evol 2006, 23:2379-2391.

22. Delorme C, Poyart C, Ehrlich SD, Renault P: Extent of horizontal gene transfer in evolution of Streptococci of the salivarius group. J Bacteriol 2007, 189:|330-134|.

23. Bolotin A, Quinquis B, Renault P, Sorokin A, Ehrlich SD, Kulakauskas S, Lapidus A, Goltsman E, Mazur M, Pusch GD, Fonstein M, Overbeek R, Kyprides N, Purnelle B, Prozzi D, Ngui K, Masuy D, Hancy F, Burteau S, Boutry M, Delcour J, Goffeau A, Hols P: Complete sequence and comparative genome analysis of the dairy bacterium Streptococcus thermophilus. Nat Biotechnol 2004, 22:1554-1558.

24. Delorme C: Streptococcus thermophilus. International Journal of Food Microbiology 1 26:274-277.

25. Holden MTG, Heather Z, Paillot R, Steward K, Webb K, Ainslie F, Jourdan T, Bason N, Holroyd N, Mungall K, Quail MA, Sanders M, Simmonds M, Willey D, Brooks K, Aanensen DM, Spratt BG, Jolley KA, Maiden MC, Kehoe M, Chanter N, Robinson C, Maskell D, Parkhill J, Waller AS: Genomic evidence for the evolution of Streptococcus equi: host restriction, increased virulence and genetic exchange with human pathogens. in press.

26. Holden MT, Scott A, Cherevach I, Chillingworth T, Churcher C, Cronin A, Dowd L, Feltwell T, Hamlin N, Holroyd S, Jagels K, Moule S, Mungall K, Quail MA, Price C, Rabbinowitsch E, Sharp S, Skelton J, Whitehead S, Barrell BG, Kehoe M, Parkhill J: Complete genome of acute rheumatic fever-associated serotype M5 Streptococcus pyogenes strain manfredo. J Bacteriol 2007, 189: I 473-I477.

27. Facklam R: What happened to the streptococci: overview of taxonomic and nomenclature changes. Clin Microbiol Rev 2002, 15:613-630.

28. Beres SB, Musser JM: Contribution of exogenous genetic elements to the group A Streptococcus metagenome. PLoS ONE 2007, 2:e800.

29. Tettelin H, Masignani V, Cieslewicz MJ, Donati C, Medini D, Ward NL, Angiuoli SV, Crabtree J, Jones AL, Durkin AS, Deboy RT, Davidsen TM, Mora M, Scarselli M, Margarit y Ros I, Peterson JD, Hauser CR, Sundaram JP, Nelson WC, Madupu R, Brinkac LM, Dodson RJ, Rosovitz MJ, Sullivan SA, Daugherty SC, Haft DH, Selengut J, Gwinn ML, Zhou L, Zafar N, Khouri H, Radune D, Dimitrov G, Watkins K, O'Connor KJ, Smith S, Utterback TR, White O, Rubens CE, Grandi G, Madoff LC, Kasper DL, Telford JL, Wessels MR, Rappuoli R, Fraser $C M$ : Genome analysis of multiple pathogenic isolates of Streptococcus agalactiae: implications for the microbial "pan-genome". Proc Natl Acad Sci USA 2005, 102: I 3950-1 3955.

30. Cantarel BL, Coutinho PM, Rancurel C, Bernard T, Lombard V, Henrissat B: The Carbohydrate-Active EnZymes database (CAZy): an expert resource for Glycogenomics. Nucleic Acids Res 2008

31. Poolman B: Transporters and their roles in LAB cell physiology. Antonie Van Leeuwenhoek 2002, 82:147-164

32. Ajdic D, McShan WM, McLaughlin RE, Savic G, Chang J, Carson MB, Primeaux C, Tian R, Kenton S, Jia H, Lin S, Qian Y, Li S, Zhu H, Najar $F$, Lai H, White J, Roe BA, Ferretti J]: Genome sequence of Streptococcus mutans UAI59, a cariogenic dental pathogen. Proc Natl Acad Sci USA 2002, 99: 14434- I4439.

33. Yamamoto $Y$, Poyart C, Trieu-Cuot P, Lamberet G, Gruss A, Gaudu P: Respiration metabolism of Group B Streptococcus is activated by environmental haem and quinone and contributes to virulence. Mol Microbiol 2005, 56:525-534.

34. Rezaiki L, Lamberet G, Derre A, Gruss A, Gaudu P: Lactococcus lactis produces short-chain quinones that cross-feed Group B Streptococcus to activate respiration growth. Mol Microbiol 2008, 67:947-957.

35. Ault-Riche D, Fraley CD, Tzeng CM, Kornberg A: Novel assay reveals multiple pathways regulating stress-induced accumulations of inorganic polyphosphate in Escherichia coli. J Bacteriol 1998, 180:1841-1847.

36. Kim KS, Rao NN, Fraley CD, Kornberg A: Inorganic polyphosphate is essential for long-term survival and virulence factors in Shigella and Salmonella spp. Proc Natl Acad Sci USA 2002, 99:7675-7680
37. Jahid IK, Silva AJ, Benitez JA: Polyphosphate stores enhance the ability of Vibrio cholerae to overcome environmental stresses in a low-phosphate environment. Appl Environ Microbiol 2006, 72:7043-7049.

38. McMeechan A, Lovell MA, Cogan TA, Marston KL, Humphrey TJ, Barrow PA: Inactivation of ppk differentially affects virulence and disrupts ATP homeostasis in Salmonella enterica serovars Typhimurium and Gallinarum. Res Microbiol 2007, I 58:79-85.

39. Candon HL, Allan BJ, Fraley CD, Gaynor EC: Polyphosphate kinase $I$ is a pathogenesis determinant in Campylobacter jejuni. J Bacteriol 2007, 189:8099-8I08.

40. Heir E, Sundheim G, Holck AL: The Staphylococcus qacH gene product: a new member of the SMR family encoding multidrug resistance. FEMS Microbiol Lett 1998, 163:49-56.

4I. Wirawan RE, Swanson KM, Kleffmann T, Jack RW, Tagg JR: Uberolysin: a novel cyclic bacteriocin produced by Streptococcus uberis. Microbiology 2007, I 53:1619-1630.

42. Wirawan RE, Klesse NA, Jack RW, Tagg JR: Molecular and genetic characterization of a novel nisin variant produced by Streptococcus uberis. Appl Environ Microbiol 2006, 72: I | 48-I I 56.

43. Heng NC, Burtenshaw GA, Jack RW, Tagg JR: Ubericin A, a class Ila bacteriocin produced by Streptococcus uberis. Appl Environ Microbiol 2007, 73:7763-7766.

44. Holden MTG, Feil EJ, Lindsay JA, Peacock SJ, Day NPJ, Enright MC, Foster TJ, Moore CE, Hurst L, Atkin R, Barron A, Bason N, Bentley SD, Chillingworth C, Chillingworth T, Churcher C, Clark L, Corton C, Cronin A, Doggett J, Dowd L, Feltwell T, Hance Z, Harris B, Hauser $\mathrm{H}$, Holroyd S, Jagels K, James KD, Lennard N, Line A, Mayes R, Moule S, Mungall K, Ormond D, Quail MA, Rabbinowitsch E, Rutherford K, Sanders M, Sharp S, Simmonds M, Stevens K, Whitehead S, Barrell BG, Spratt BG, Parkhill J: Complete genomes of two clinical Staphylococcus aureus strains: Evidence for the rapid evolution of virulence and drug resistance. Proc Natl Acad Sci USA 2004, I $01: 9786-9791$.

45. Lerat $\mathrm{E}$, Ochman $\mathrm{H}$ : Recognizing the pseudogenes in bacterial genomes. Nucleic Acids Res 2005, 33:3125-3132.

46. Christie J, McNab R, Jenkinson HF: Expression of fibronectinbinding protein FbpA modulates adhesion in Streptococcus gordonii. Microbiology 2002, 148:1615-1625.

47. Comfort D, Clubb RT: A comparative genome analysis identifies distinct sorting pathways in gram-positive bacteria. Infect Immun 2004, 72:2710-2722.

48. Dramsi S, Trieu-Cuot P, Bierne H: Sorting sortases: a nomenclature proposal for the various sortases of Gram-positive bacteria. Res Microbiol 2005, I 56:289-297.

49. Pallen MJ, Lam AC, Antonio M, Dunbar K: An embarrassment of sortases - a richness of substrates? Trends Microbiol 200I, 9:97-102.

50. Mazmanian SK, Liu G, Ton-That H, Schneewind O: Staphylococcus aureus sortase, an enzyme that anchors surface proteins to the cell wall. Science 1999, 285:760-763.

51. Schneewind O, Mihaylova-Petkov D, Model P: Cell wall sorting signals in surface proteins of gram-positive bacteria. Embo J 1993, I 2:4803-48। I.

52. Moshynskyy I, Jiang M, Fontaine MC, Perez-Casal J, Babiuk LA, Potter AA: Characterization of a bovine lactoferrin binding protein of Streptococcus uberis. Microb Pathog 2003, 35:203-2I5.

53. Whatmore AM: Streptococcus pyogenes sclB encodes a putative hypervariable surface protein with a collagen-like repetitive structure. Microbiology 200I, 147:419-429.

54. Hagiwara S, Kawai K, Anri A, Nagahata H: Lactoferrin concentrations in milk from normal and subclinical mastitic cows. J Vet Med Sci 2003, 65:319-323.

55. Harmon RJ, Schanbacher FL, Ferguson LC, Smith KL: Changes in lactoferrin, immunoglobulin G, bovine serum albumin, and alpha-lactalbumin during acute experimental and natural coliform mastitis in cows. Infect Immun 1976, I3:533-542.

56. Todhunter DA, Smith KL, Schoenberger PS: In vitro growth of mastitis-associated streptococci in bovine mammary secretions. J Dairy Sci 1985, 68:2337-2346.

57. Almeida RA, Luther DA, Park HM, Oliver SP: Identification, isolation, and partial characterization of a novel Streptococcus uberis adhesion molecule (SUAM). Vet Microbiol 2006, II5:183-19I. 
58. Fang W, Oliver SP: Identification of lactoferrin-binding proteins in bovine mastitis-causing Streptococcus uberis. FEMS Microbiol Lett 1999, I 76:91-96.

59. Fang W, Almeida RA, Oliver SP: Effects of lactoferrin and milk on adherence of Streptococcus uberis to bovine mammary epithelial cells. Am J Vet Res 2000, 6 I:275-279.

60. Chen CC, Cleary PP: Complete nucleotide sequence of the streptococcal C5a peptidase gene of Streptococcus pyogenes. J Biol Chem 1990, 265:316I-3167.

61. Hollingshead SK, Fischetti VA, Scott JR: Complete nucleotide sequence of type $6 \mathrm{M}$ protein of the group A Streptococcus. Repetitive structure and membrane anchor. J Biol Chem 1986, 26I:I677-I686.

62. Martin PR, Hoiby EA: Streptococcal serogroup A epidemic in Norway 1 987-1 988. Scand J Infect Dis 1990, 22:42 I-429.

63. Cho KH, Caparon MG: Patterns of virulence gene expression differ between biofilm and tissue communities of Streptococcus pyogenes. Mol Microbiol 2005, 57:I545-I556.

64. Ward PN, Field TR, Ditcham WG, Maguin E, Leigh JA: Identification and disruption of two discrete loci encoding hyaluronic acid capsule biosynthesis genes has $A$, hasB, and has $C$ in Streptococcus uberis. Infect Immun 200I, 69:392-399.

65. Dougherty BA, Rijn I van de: Molecular characterization of hasA from an operon required for hyaluronic acid synthesis in group A streptococci. J Biol Chem 1994, 269:169-175

66. Ashbaugh CD, Alberti S, Wessels MR: Molecular analysis of the capsule gene region of group $A$ Streptococcus: the has $A B$ genes are sufficient for capsule expression. J Bacteriol 1998 , I 80:4955-4959.

67. Field TR, Ward PN, Pedersen LH, Leigh JA: The hyaluronic acid capsule of Streptococcus uberis is not required for the development of infection and clinical mastitis. Infect Immun 2003 , 71:132-139.

68. Rosey EL, Lincoln RA, Ward PN, Yancey RJ Jr, Leigh JA: PauA: a novel plasminogen activator from Streptococcus uberis. FEMS Microbiol Lett 1999, I 78:27-33.

69. Ward PN, Leigh JA: Characterization of PauB, a novel broad spectrum plasminogen activator from Streptococcus uberis. J Bacteriol 2002, I 84: I 19-125

70. Malke H, Steiner K, Gase K, Frank C: Expression and regulation of the streptokinase gene. Methods 2000, 2 I: I I I-I 24.

7I. Mechold U, Steiner K, Vettermann S, Malke H: Genetic organization of the streptokinase region of the Streptococcus equisimilis H46A chromosome. Mol Gen Genet 1993, 24 I: I 29- I40.

72. Canchaya C, Desiere F, McShan WM, Ferretti J], Parkhill J, Brussow $\mathrm{H}$ : Genome analysis of an inducible prophage and prophage remnants integrated in the Streptococcus pyogenes strain SF370. Virology 2002, 302:245-258.

73. Banks DJ, Porcella SF, Barbian KD, Beres SB, Philips LE, Voyich JM, DeLeo FR, Martin JM, Somerville GA, Musser JM: Progress toward characterization of the group A Streptococcus metagenome: complete genome sequence of a macrolide-resistant serotype M6 strain. J Infect Dis 2004, 190:727-738.

74. Starr CR, Engleberg NC: Role of hyaluronidase in subcutaneous spread and growth of group A streptococcus. Infect Immun 2006, 74:40-48.

75. Feldman C, Cockeran R, Jedrzejas MJ, Mitchell TJ, Anderson R: Hyaluronidase augments pneumolysin-mediated injury to human ciliated epithelium. Int I Infect Dis 2007, I I: I I- I 5.

76. Christie R, Atkins NE, Munch-Petersen E: A note on a lytic phenomenon shown by group B streptococci. Aust J Exp Biol Med Sci 1944, 22: 197-200.

77. Jiang $M$, Babiuk LA, Potter AA: Cloning, sequencing and expression of the CAMP factor gene of Streptococcus uberis. Microb Pathog 1996, 20:297-307.

78. Hill AW, Leigh JA: DNA fingerprinting of Streptococcus uberis: a useful tool for epidemiology of bovine mastitis. Epidemio Infect 1989, 103:165-I7I.

79. Rutherford K, Parkhill J, Crook J, Horsnell T, Rice P, Rajandream MA, Barrell B: Artemis: sequence visualization and annotation. Bioinformatics 2000, 16:944-945.

80. Frishman D, Mironov A, Mewes HW, Gelfand M: Combining diverse evidence for gene recognition in completely sequenced bacterial genomes. Nucleic Acids Res 1998, 26:294I-2947.
81. Delcher AL, Harmon D, Kasif S, White O, Salzberg SL: Improved microbial gene identification with GLIMMER. Nucleic Acids Res 1999, 27:4636-464l.

82. Larsen TS, Krogh A: EasyGene-a prokaryotic gene finder that ranks ORFs by statistical significance. BMC Bioinformatics 2003, 4:21.

83. Altschul SF, Gish W, Miller W, Myers EW, Lipman DJ: Basic loca alignment search tool. J Mol Biol 1990, 2 I 5:403-4 I0.

84. Pearson WR, Lipman DJ: Improved tools for biological sequence comparison. Proc Natl Acad Sci USA 1988, 85:2444-2448.

85. Bateman A, Birney E, Cerruti L, Durbin R, Etwiller L, Eddy SR, Griffiths-Jones S, Howe KL, Marshall M, Sonnhammer EL: The Pfam protein families database. Nucleic Acids Res 2002, 30:276-280.

86. Falquet L, Pagni M, Bucher P, Hulo N, Sigrist CJ, Hofmann K, Bairoch A: The PROSITE database, its status in 2002. Nucleic Acids Res 2002, 30:235-238.

87. Krogh A, Larsson B, von Heijne G, Sonnhammer EL: Predicting transmembrane protein topology with a hidden Markov model: application to complete genomes. J Mol Biol 200I, 305:567-580

88. Nielsen H, Engelbrecht J, Brunak S, von Heijne G: Identification of prokaryotic and eukaryotic signal peptides and prediction of their cleavage sites. Protein Eng 1997, 10:1-6.

89. Lowe TM, Eddy SR: tRNAscan-SE: a program for improved detection of transfer RNA genes in genomic sequence. Nucleic Acids Res 1997, 25:955-964.

90. Griffiths-Jones S, Bateman A, Marshall M, Khanna A, Eddy SR: Rfam: an RNA family database. Nucleic Acids Res 2003, 3 I:439-44I.

91. Carver T], Rutherford KM, Berriman M, Rajandream MA, Barrell BG, Parkhill J: ACT: the Artemis Comparison Tool. Bioinformatics 2005, 2 I :3422-3423.

92. Beres SB, Sylva GL, Barbian KD, Lei BF, Hoff JS, Mammarella ND, Liu MY, Smoot JC, Porcella SF, Parkins LD, Campbell DS, Smith TM, McCormick JK, Leung DYM, Schlievert PM, Musser JM: Genome sequence of a serotype M3 strain of group A Streptococcus: Phage-encoded toxins, the high-virulence phenotype, and clone emergence. Proc Natl Acad Sci USA 2002, 99: I0078-10083.

93. Holden MTG, Sanders M, Hauser H, Hoa NT, Cherevach I, Chillingworth T, Churcher C, Cronin A, Dowd L, Feltwell T, Hamlin N, Holroyd $\mathrm{N}$, Jagels $\mathrm{K}$, Moule $\mathrm{S}$, Mungall $\mathrm{K}$, Quail MA, Price $\mathrm{C}$, Rabbinowitsch E, Sharp S, Skelton J, Whitehead S, Croucher NJ, Kehoe M, Leigh J, Ward NL, Dowson C, Whatmore AM, Chanter N, Iversen P, Gottschalk M, Slater J, Smith HO, Spratt B, Xe J, Ye C Bentley SD, Barrell BG, Schultsz C, Maskell D, Parkhill J: Evidence for the rapid evolution of virulence and drug resistance in the zoonotic pathogen Streptococcus suis. in press.

94. Tettelin H, Nelson KE, Paulsen IT, Eisen JA, Read TD, Peterson S, Heidelberg J, DeBoy RT, Haft DH, Dodson RJ, Durkin AS, Gwinn M, Kolonay JF, Nelson WC, Peterson JD, Umayam LA, White O, Salzberg SL, Lewis MR, Radune D, Holtzapple E, Khouri H, Wolf AM, Utterback TR, Hansen CL, McDonald LA, Feldblyum TV, Angiuoli S, Dickinson T, Hickey EK, Holt IE, Loftus BJ, Yang F, Smith HO, Venter IC, Dougherty BA, Morrison DA, Hollingshead SK, Fraser CM: Complete genome sequence of a virulent isolate of Streptococcus pneumoniae. Science 200I, 293:498-506.

95. Xu P, Alves JM, Kitten T, Brown A, Chen Z, Ozaki LS, Manque P, Ge X, Serrano MG, Puiu D, Hendricks S, Wang Y, Chaplin MD, Akan D, Paik S, Peterson DL, Macrina FL, Buck GA: Genome of the opportunistic pathogen Streptococcus sanguinis. I Bacteriol 2007, I 89:3 | 66-3175

96. Glaser P, Rusniok C, Buchrieser C, Chevalier F, Frangeul L, Msadek T, Zouine M, Couve E, Lalioui L, Poyart C, Trieu-Cuot P, Kunst F: Genome sequence of Streptococcus agalactiae, a pathogen causing invasive neonatal disease. Mol Microbiol 2002 , 45: $1499-1513$

97. Vickerman MM, lobst S, Jesionowski AM, Gill SR: Genome-wide transcriptional changes in Streptococcus gordonii in response to competence signaling peptide. J Bacteriol 2007, | 89:7799-7807.

98. Bolotin A, Wincker $\mathrm{P}$, Mauger S, Jaillon $\mathrm{O}$, Malarme $\mathrm{K}$, Weissenbach J, Ehrlich SD, Sorokin A: The complete genome sequence of the lactic acid bacterium Lactococcus lactis ssp. lactis ILI403. Genome Res 2001, I I:731-753.

99. Guindon S, Gascuel O: A simple, fast, and accurate algorithm to estimate large phylogenies by maximum likelihood. Syst Biol 2003, 52:696-704. 
100. Guindon S, Lethiec F, Duroux P, Gascuel O: PHYML Online-a web server for fast maximum likelihood-based phylogenetic inference. Nucleic Acids Res 2005, 33:W557-559.

101. Perriere G, Gouy M: WWW-query: an on-line retrieval system for biological sequence banks. Biochimie 1996, 78:364-369.

102. Thompson JD, Gibson TJ, Plewniak F, Jeanmougin F, Higgins DG: The CLUSTAL_X windows interface: flexible strategies for multiple sequence alignment aided by quality analysis tools. Nucleic Acids Res 1997, 25:4876-4882.

Publish with Biomed Central and every scientist can read your work free of charge

"BioMed Central will be the most significant development for disseminating the results of biomedical research in our lifetime. "

Sir Paul Nurse, Cancer Research UK

Your research papers will be:

- available free of charge to the entire biomedical community

- peer reviewed and published immediately upon acceptance

- cited in PubMed and archived on PubMed Central

- yours - you keep the copyright 